\title{
Intestinal parasitosis in a population of foreign subjects of recent immigration
}

\author{
Carlotta Guidetti', Lidia Ricci', Luigi Vecchia² \\ I L.P. in Microbiologia Virologia \\ 2 Laboratorio Analisi Chimico-Cliniche e di Endocrinologia, Arcispedale Santa Maria Nuova, Reggio Emilia
}

Key words: Bowel helminths, Bowel protozoa, Diagnosis, Migrants, Parasitosis

\section{Parassitosi intestinali in una popolazione di soggetti stranieri di recente immigrazione}

\section{SUMMARY}

The purpose of this survey is to assess the prevalence of intestinal parasites in a population of subjects recently immigrated and hosted in the Center for the Foreign Family Health in Reggio Emilia, they are recent immigrants without valid residence permit. 160 faecal samples were collected together with peripheral blood samples of the same subjects enrolled in the study with the random methodology for a period of about 4 months after completion of a cognitive-anamnestic cards. A copro-parassitological standard examination, blood count and platelets count were performed.Among the subjects enrolled and who have joined the project, nearly $50 \%$ tested positive for one or more pests in the copro-parasitological exams. The species most represented were the protozoa with prevalence of Blastocystis hominis and Dientamoeba fragilis, in respect of Entamoeba coli and Giardia duodenalis; helminths were rarely observed and Hymenolepis nana, Schistosoma haematobium, and Ascaris lumbricoides were observed. The analysis of cognitive-anamnestic cards showed that about half of the parasitized individuals show no clinical symptoms that could be traced to the infection / infestation of parasitic type. The result of this epidemiological survey shows the need to pay more attention to the immigrants as potential carriers of important parasitic infections.

\section{INTRODUZIONE}

Le parassitosi intestinali, sostenute sia da protozoi che elminti, sono patologie che interessano tutte le regioni del globo, sebbene la massima diffusione si abbia nei Paesi in via di sviluppo, a causa della presenza di svariati fattori: climi caldi e caldoumidi, elevata densità di popolazione, scarse o scarsissime condizioni igieniche, presenza di insetti vettori o veicoli passivi di parassiti, poche risorse economiche a disposizione per intervenire al riguardo, usi e costumi (alimentari e non) delle popolazioni medesime (13).

In Italia le parassitosi autoctone sembrano essere attualmente piuttosto rare $(3,11,12)$, soprattutto in virtù degli elevati standard igienici ormai raggiunti più o meno ovunque e delle adeguate abitudini idro-alimentari oggi esistenti nel nostro Paese $(1,7,11)$. I crescenti flussi migratori da Paesi in via di sviluppo tecnologico (soprattutto da paesi africani, asiatici, Europa dell'est, del centro e sudAmerica) hanno fatto riemergere patologie ormai quasi scomparse nel nostro paese. Si tratta per lo più di malattie a trasmissione sessuale o tubercolosi polmonare; tuttavia anche le parassitosi intestinali, laddove siano diagnosticate, non sono affatto rare in questa tipologia di persone $(11,12$, $23,26)$. Scopo del presente studio è quello di valutare la prevalenza di parassitosi intestinali di importazione nella provincia di Reggio Emilia, attraverso uno studio prospettico che ha coinvolto una ristretta popolazione di stranieri, comunitari ed extracomunitari, di recente immigrazione, arrivati nella nostra provincia in condizioni di clandestinità. L'idea dello studio epidemiologico descritto nasce dall'analisi dei risultati ottenuti in una indagine da noi effettuata presso il Laboratorio di Microbiologia dell'Arcispedale Santa Maria Nuova di Reggio Emilia (19), in cui è emerso che le parassitosi intestinali di importazione sono di gran lunga superiori a quelle strettamente autoctone. Abbiamo applicato metodologie diagnostiche raccomandate dalle linee guida nazionali e internazionali, raccolto il maggior numero possibile di dati anamnestici relativi ai soggetti sottoposti ad indagine parassitologica, al fine di essere in grado di fornire informazioni riguardo i parassiti identificati, la provenienza dei soggetti maggiormente parassitati e stabilire correlazioni tra le diverse specie di parassita e le diverse sintomatologie cliniche ad essi correlati o correlabili $(4,13,14)$.

\section{MATERIALI E METODI}

L'arruolamento dei soggetti è stato eseguito presso il "Centro per la Salute della Famiglia

\section{Corresponding author: Carlotta Guidetti}

L. P. in Microbiologia e Virologia

Via Gramsci, 2 - 42019 Scandiano (RE) - Cell.: 3498466603

E-mail: carlottaguidetti@libero.it 
Straniera" di Reggio Emilia, poliambulatorio deputato alla tutela della salute della popolazione immigrata senza regolare permesso di soggiorno. Nel periodo che va da Aprile a Luglio 2009, ai soggetti che afferivano al centro è stato spiegato lo scopo dell'indagine, che consisteva nella valutazione epidemiologico - conoscitiva delle eventuali parassitosi nei soggetti stessi, tali da potere essere interpretabili come parassitosi intestinali di importazione. Il criterio di arruolamento è stato del tutto casuale, riguardo l'etnia di appartenenza, l'età, il sesso, pur privilegiando, per quanto possibile, i soggetti di più recente immigrazione $(<2$ anni). A coloro che hanno aderito al progetto è stata fatta compilare una scheda anamnestico conoscitiva in cui oltre ai dati anagrafici veniva richiesta la zona di provenienza, il tempo di immigrazione e informazioni riguardo la sintomatologia clinica (quando presente).

E stato inoltre eseguito un prelievo venoso per l'esecuzione di un emocromo con lo scopo di valutare l'eventuale ipereosinofilia spesso correlata a un'infezione/infestazione di tipo parassitologico $(10,12)$; ai soggetti è stato consegnato, infine, un contenitore sterile (con le opportune istruzioni circa la modalità di raccolta del campione fecale) da restituire poi nei giorni successivi.

I soggetti arruolati sono stati 200 , ma coloro che hanno consegnato il campione fecale, consentendo così il completamento dello studio, sono stati 160 (pari all' $80 \%$ ). Per ogni soggetto che ha aderito allo studio è stato analizzato un solo campione fecale $(5,6)$. Non erano comprese nello studio né la ricerca mirata di ossiuri (Enterobius vermicularis), per la ricerca delle cui uova è necessario ricorrere allo scotch test, né la ricerca mirata di Strongyloides stercoralis (4). Dei 160 soggetti che hanno aderito allo studio, 44 erano bambini (da 0 a 14 anni) e 116 erano adulti (15- 76 anni); 83 erano maschi e 77 erano femmine; 89 provenivano dal continente Africano, 38 dalle zone dell'est europeo, 30 dal continente Asiatico e 3 dall'America meridionale; 49 erano immigrati in Italia da un periodo inferiore ad 1 mese, 36 da 1 a 6 mesi, 38 da 7 mesi a 1 anno, 23 da 1 a 3 anni e 34 dimoravano nel nostro Paese da più di 3 anni. L'analisi dei campioni fecali è stata eseguita mediante esame copro-parassitologico standard (ECPS) cercando di aderire il più possibile alle metodologie diagnostiche raccomandate in letteratura $(4,8,16,17,18,22,27,28,31,32)$. L'ECPS prevede: esame macroscopico a fresco del campione fecale (per la ricerca di eventuali proglottidi di tenie o vermi adulti e per una valutazione della consistenza del campione); esame microscopico diretto a fresco (per la ricerca di trofozoiti di amebe o di altri protozoi), utilizzazione del metodo ICT: "Triage ${ }^{\circledR}$ parasite panel" (biosite Diagnostics), colorazione permanente con Giemsa per la ricerca principalmente di Dientamoeba fragilis ed esame microscopico dopo arricchimento formolo-etilacetato (FEA: per la ricerca di uova di elminti, cisti di Giardia duodenalis e dei principali protozoi non patogeni). Il FEA è stato eseguito con il Kit: PARA-PAK PLUS SAF (Meriadian Bioscience Europa).

\section{RISULTATI}

Dei 160 soggetti che hanno aderito allo studio, quelli affetti da una parassitosi intestinale sono risultati 77 , pari al $48.1 \%$ : in 21 casi, pari al $13.1 \%$ sono stati reperiti parassiti patogeni e in 56 casi, pari al 35\% del totale, sono stati reperiti parassiti non patogeni (Figura I).

Dei 77 soggetti positivi, 29, pari al $37.7 \%$ erano bambini (12 maschi e 17 femmine) e 48, pari al $62.3 \%$, erano adulti (31 maschi e 17 femmine); il $67.5 \%$ provenivano dal continente africano (Egitto, Sahrawi, Tunisia, Marocco, Burkina Faso, Ghana, Senegal e Nigeria); il 23.4\% dall'Europa (Romania, Moldavia, Georgia, Ucraina, Bosnia e Albania); il $6.5 \%$ e il $2.6 \%$ rispettivamente da Asia (Cina, Vietnam, Pakistan e Palestina) e America del sud (Ecuador e Uruguay).

Le positività correlate al tempo di immigrazione sono riassunte in Tabella 1. Tra i soggetti arrivati nel nostro paese da meno di un mese è risultato positivo il $63.2 \%$; analoga situazione per coloro che dimoravano in Italia da un periodo compreso tra 1 e 6 mesi, tra cui è risultato positivo il $62.5 \%$. Le percentuali di positività tendono invece a diminuire tra $\mathrm{i}$ soggetti immigrati da 1 a 3 anni $(52.1 \%)$ e si riducono drasticamente nei soggetti che hanno lasciato i paesi di origine da più di 3 anni $(11.7 \%)$.

In Tabella 2 sono riportate le prevalenze di parassitosi intestinali in base alla patogenicità della specie parassitaria: il $20.4 \%$ dei bambini è risultato positivo per parassiti patogeni, mentre il $45.4 \%$ per parassiti non patogeni. Negli adulti sono risultati positivi per patogeni il $10.3 \%$ e per non patogeni il $31.0 \%$.

Per quanto riguarda la prevalenza di parassitosi in base al sesso, il $15 \%$ dei maschi e il $25 \%$ delle femmine in età pediatrica sono risultati positivi per protozoi patogeni o elminti (sempre considerati patogeni), mentre le positività per parassiti non patogeni sono molto simili in entrambi i sessi: $45.0 \%$ nei bambini e $45.8 \%$ nelle bambine.

Il $12.7 \%$ dei maschi adulti erano positivi per patogeni e il $36.5 \%$ per non patogeni. Nella popolazione adulta femminile, le prevalenze sono state del $7.5 \%$ per patogeni e del $24.5 \%$ per non patogeni. 
In Tabella 3 sono riportate la distribuzione e la quantificazione delle parassitosi osservate. Sono stati riscontrati soggetti poliparassitati, per cui su 77 soggetti positivi i parassiti reperiti nei campioni fecali sono stati 106 .

Trentanove soggetti, pari al $24.5 \%$ del totale, erano parassitati da un solo protozoo non patogeno (19 Blastocystis hominis; 2 Entamoeba histolytical dispar classificata tra i non patogeni per l'assenza di sintomatologia specifica e di trofozoiti con fenomeni di fagocitosi a carico di emazie; 7 Endolimax nana, 11 Entamoeba coli) e 7, pari al $4.4 \%$ del totale, erano parassitati da un solo protozoo patogeno (4 Giardia duodenalis e 3 Dientamoeba fragilis); in 5 soggetti è stato reperito un solo elminta (2 Hymenolepis nana, 1 Schistosoma haematobium, 1 Ancylostoma duodenale/Necator americanus, 1 Ascaris lubricoides) e nei 26 soggetti poliparassitati sono stati reperiti protozoi non patogeni in associazione tra di loro $(10 \%)$ o associati con patogeni $(6.3 \%)$ : (5 Blastocystis hominis + Endolimax nana; 2 Blastocystis hominis + Endolimax nana + Entamoeba coli; 3 Blastocystis hominis + Entamoeba coli; 5 Entamoeba coli + Endolimax nana; 1 Entamoeba coli + Entamoeba hartmanni; 3 Blastocystis hominis + Giardia duodenalis; 1 Blastocystis hominis + Entamoeba coli + Hymenolepis nana; 2 Blastocystis hominis + Dientamoeba fragilis; 1 Entamoeba coli + Dientamoeba fragilis; 1 Entamoeba coli + Giardia duodenalis; 1 Entamoeba coli + Giardia duodenalis + Endolimax nana; 1 Endolimax nana + Dientamoeba fragilis).

Per quanto riguarda la sintomatologia clinica sono state analizzate le schede clinico - anamnestiche conoscitive relative ai soli soggetti infestati da almeno un parassita (patogeno o meno).

In Tabella 4 sono riportate le correlazioni cliniche generali con i parassiti osservati.

Per ipereosinofilia si intende una percentuale di eosinofili circolanti superiore al 6\% (> 500 eosinofili/microlitro). Per disturbi intestinali aspecifici si intende il perpetuarsi di sensazione di nausea e vomito e, per quanto riguarda le diarree, sono state prese in considerazione sia diarree acute che protratte nel tempo.

Circa la metà delle infezioni/infestazioni parassitarie decorre in maniera sintomatica; le sintomatologie più frequentemente riscontrate sono: disturbi addominali aspecifici, correlabili alla presenza di infezioni protozoarie e ipereosinofilia periferica, solitamente correlata alla presenza di elminti. In Tabella 5 sono illustrate le correlazioni tra parassiti e consistenza del campione fecale.

In generale la maggior parte delle parassitosi, sia sostenute da patogeni che da non patogeni, non correlano con la consistenza liquida delle feci. Circa $1^{6} 80 \%$ degli elminti e dei protozoi è stato repertato in campioni di aspetto solido o semisolido.

\section{DISCUSSIONE}

Analizzando i risultati, ci troviamo di fronte a percentuali di positività ben più elevate rispetto a quelle che normalmente caratterizzano gli studi condotti sulle popolazioni autoctone nelle diverse realtà italiane $(2,9,14,15,19,23,26)$, dal momento che la popolazione in esame è costituita da gruppi di persone socialmente disagiate, in condizioni di clandestinità e per la maggior parte senza una fissa dimora (23).

Le percentuali di positività, tuttavia, subiscono una notevole diminuzione mano a mano che si prolunga il tempo di permanenza nel nostro paese passando da un $63.2 \%$ a un $11.7 \%$.

Questo dato dimostra che trascorso un certo lasso di tempo ed in seguito al variare delle condizioni ambientali, buona parte di questi soggetti tende a perdere lo stato di soggetto parassitato.

Per quanto riguarda gli agenti eziologici, tra i protozoi patogeni, le specie maggiormente rappresentate sono: Giardia duodenalis e Dientamoeba fragilis.

Gli elminti sono stati identificati in soggetti di recentissima immigrazione: gli unici due casi di geoelmintiasi sostenute dai nematodi Ascaris lumbricoides (Figura II) e Ancylostoma duodenalel Necator americanus (Figura III) sono stati riscontrati rispettivamente in un bambino adottato ed in un ragazzo Pakistano arrivati nel nostro paese da soli 10 giorni; tra i trematodi è stato reperito un solo Schistosoma haematobium (Figura IV) nel campione fecale di una ragazza proveniente dal Burkina Faso, residente in Italia da due anni ma rientrante da un soggiorno nel paese di origine.

Tra i cestodi, le tre infestazioni da Hymenolepis nana riguardano due bambini provenienti dal Sahrawi arrivati in Italia da pochi giorni per un soggiorno estivo nel nostro paese e un bambino rumeno residente in un campo nomadi nella provincia di Reggio Emilia, arrivato in Italia tre settimane prima dell'esecuzione dell'esame coproparassitologico.

Per quanto concerne i protozoi non patogeni rilevati, nella maggior parte dei casi si trattava di Blastocystis hominis, seguita da Entamoeba coli ed Endolimax nana; Entamoeba hartmanni e Chilomastix mesnili sono state reperite in un numero esiguo di casi. Entamoeba histolytica/dispar, riscontrata in 2 soggetti è stata diagnosticata all'esame microscopico e la sua presenza confermata col metodo immunocromatografico (Triage ${ }^{\circledR}$ parasite panel Biosite HQ and Manufacturing 
Campus, Mera Misa, California, USA). In assenza di sintomatologia specifica e di trofozoiti con fenomeni di fagocitosi a carico di emazie, $E$. histolytica/dispar è stata classificata tra i protozoi non patogeni. Vanno precisate frequenti associazioni osservate soprattutto tra parassiti patogeni e non patogeni; i parassiti riscontrati negli 77 soggetti positivi sono stati 106 e le associazioni sono state osservate in 21 soggetti (27.2\%).

Per quanto concerne gli aspetti clinici, sulla base delle schede anamnestiche, emerge che la sintomatologia, non sempre presente, è la più variegata possibile, come atteso e prevedibile (11).

Per quanto riguarda le elmintiasi, il 50\% dei soggetti infestati presentava ipereosinofilia periferica. Tra i protozoi patogeni Dientameoba fragilis è, nell'indagine riportata, il parassita che mag-

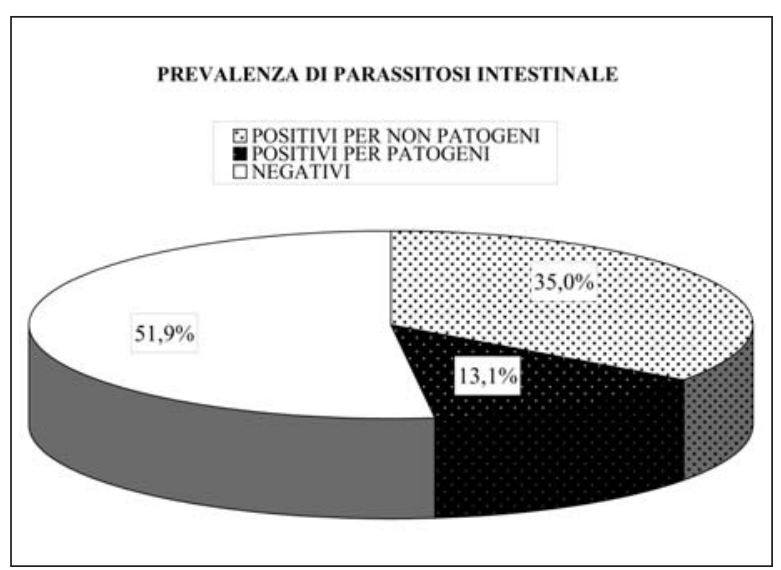

Figura I. Percentuali di positività per parassitosi intestinale sostenuta da patogeni e non patogeni.

Tabella I. Percentuali di positività calcolate in base al tempo di immigrazione

TEMPO DI IMMIGRAZIONE POSTIVO/TOTALE

\begin{tabular}{ccc}
\hline$<$ di I mese & $3 / / 49$ & $\mathbf{6 3 . 2} \%$ \\
\hline Da I a 6 mesi & $10 / 16$ & $\mathbf{6 2 . 5 \%}$ \\
\hline Da 7 mesi a I anno & $20 / 38$ & $\mathbf{5 2 . 6 \%}$ \\
\hline Da I a 3 anni & $12 / 23$ & $\mathbf{5 2 . 1 \%}$ \\
\hline > di 3 anni & $4 / 34$ & $\mathbf{I I . 7 \%}$ \\
\hline
\end{tabular}

giormente causa disturbi addominali $(71.4 \%$ dei casi) $(10,11,20,21,24,30)$.

La sua presenza è, inoltre, a volte correlata ad un'ipereosinofilia periferica $(42.8 \%$ dei casi) $(11,33)$.

Gran parte delle giardiasi decorre in modo asintomatico $(55.5 \%)$.

Tra i protozoi non patogeni, è opportuno riflettere sul fatto che il saprofita opportunista $B$. hominis, il cui ruolo patogeno è tuttora controverso $(6,25)$, sia responsabile di dolori addominali nel $40 \%$ dei soggetti con positività per tale protozoo.

In generale è interessante notare come le parassitosi sostenute sia da patogeni che da non patogeni decorrono in maniera asintomatica in circa il 50\% dei soggetti parassitati.

Per concludere, da quanto sinora esposto, si può sottolineare che le parassitosi intestinali, soprattutto di importazione, non sono rare, e possono essere responsabili di infezioni in sede enterica in modo più frequente di alcuni più noti agenti batterici (11).

Risulta pertanto necessario sensibilizzare i medici di base, gli igienisti, i clinici dei reparti ospedalieri, in particolare quelli di medicina generale, al campo della parassitologia e alla sua diagnostica, ancora sottovalutati.

È inoltre, necessario impostare nei settori di parassitologia diagnostica un'attività operativa organizzata e ragionata, affinché siano applicate tutte le metodologie diagnostiche disponibili e percorsi codificati, secondo le linee guida ufficiali $(4,12,22,31,32)$.

Sarebbe inoltre opportuno inserire nei protocolli di screening dei servizi di igiene pubblica il test parassitologico nei soggetti arrivati clandestinamente nel nostro paese, poiché potenzialmente infetti da parassitosi importanti e, non avendo solitamente una sintomatologia clinica correlata, possono sfuggire a controlli sanitari, con conseguente potenziale diffusione incontrastata di alcuni parassiti in ambienti comunitari $(13,23)$

Tabella 2. Prospetto complessivo di prevalenza di parassitosi intestinali nei soggetti arruolati

\begin{tabular}{|c|c|c|c|c|c|c|}
\hline POSITIVI & \multicolumn{2}{|c|}{ BAMBINI } & \multicolumn{2}{|c|}{ ADULTI } & \multicolumn{2}{|c|}{ TOTALE } \\
\hline \multirow{5}{*}{$\begin{array}{c}\text { ELMINTI E PROTOZOI } \\
\text { PATOGENI }\end{array}$} & \multicolumn{2}{|c|}{$9 / 44$} & \multicolumn{2}{|c|}{$12 / 116$} & \multicolumn{2}{|c|}{$21 / 160$} \\
\hline & \multicolumn{2}{|c|}{$20.4 \%$} & \multicolumn{2}{|c|}{$10.3 \%$} & \multicolumn{2}{|c|}{$13.1 \%$} \\
\hline & $M$ & $\mathrm{~F}$ & $M$ & $\mathrm{~F}$ & $M$ & $F$ \\
\hline & $3 / 20$ & $6 / 24$ & $8 / 63$ & $4 / 53$ & $11 / 83$ & $10 / 77$ \\
\hline & $15.0 \%$ & $25.0 \%$ & $12.7 \%$ & $7.5 \%$ & $13.2 \%$ & $12.9 \%$ \\
\hline & \multicolumn{2}{|c|}{ BAMBINI } & \multicolumn{2}{|c|}{ ADULTI } & \multicolumn{2}{|c|}{ TOTALE } \\
\hline & \multicolumn{2}{|c|}{$20 / 44$} & \multicolumn{2}{|c|}{$36 / 116$} & \multicolumn{2}{|c|}{$56 / 160$} \\
\hline PROTOZOI & \multicolumn{2}{|c|}{$45.4 \%$} & \multicolumn{2}{|c|}{$31.0 \%$} & \multicolumn{2}{|c|}{$35.0 \%$} \\
\hline \multirow[t]{3}{*}{ NON PATOGENI } & $M$ & $F$ & $M$ & $\mathrm{~F}$ & $M$ & $\mathrm{~F}$ \\
\hline & $9 / 20$ & $1 \mathrm{I} / 24$ & $23 / 63$ & $13 / 53$ & $32 / 83$ & $24 / 77$ \\
\hline & $45.0 \%$ & $45.8 \%$ & $36.5 \%$ & $24.5 \%$ & $38.5 \%$ & $31.1 \%$ \\
\hline
\end{tabular}


Tabella 3. Distribuzione e prevalenza di parassitosi nella popolazione esaminata.

SOGGETTI POSITIVI

I solo protozoo non patogeno

(24.5\%)

I solo protozoo patogeno

(4.4\%)

I solo elminta

(3.1\%)

\section{2 o più protozoi non patogeni}

(10.0\%)
PARASSITA

\begin{tabular}{ccc} 
Blastocystis hominis & 19 & $\mathbf{I I . 9 \%}$ \\
\hline Entamoeba histolyticaldispar & & $1.3 \%$ \\
\hline Endolimax nana & 2 & $1.3 \%$ \\
\hline Entamoeba coli & 7 & $\mathbf{4 . 4 \%}$ \\
\hline Garda & 11 & $\mathbf{6 . 9 \%}$
\end{tabular}

Giardia duodenalis

Dientamoeba fragilis

Hymenolepis nana

Schistosoma haematobium

Ancylostoma duodenale

Ascaris lumbricoides

\begin{tabular}{ccc} 
Hymenolepis nana & 2 & $\mathbf{I . 3 \%}$ \\
\hline Schistosoma haematobium & $\mathrm{I}$ & $\mathbf{0 . 6 \%}$ \\
\hline Ancylostoma duodenale & $\mathrm{I}$ & $\mathbf{0 . 6 \%}$ \\
\hline Ascaris lumbricoides & $\mathrm{I}$ & $\mathbf{0 . 6 \%}$ \\
\hline Blastocystis hominis + Endolimax nana & 5 & $\mathbf{3 . 1 \%}$ \\
\hline Blastocystis hominis + Endolimax nana + Entamoeba coli & 2 & $\mathbf{1 . 3 \%}$ \\
\hline Blastocystis hominis + Entamoeba coli & 3 & $\mathbf{1 . 9 \%}$ \\
\hline Entamoeba coli + Endolimax nana & 5 & $\mathbf{3 . 1 \%}$ \\
\hline Entamoeba coli + Entamoeba hartmanni & $\mathrm{I}$ & $\mathbf{0 . 6 \%}$ \\
\hline Blastocystis hominis + Giardia duodenalis & 3 & $\mathbf{1 . 9 \%}$ \\
\hline Blastocystis hominis + Entamoeba coli + Hymenolepis nana & $\mathrm{I}$ & $\mathbf{0 . 6 \%}$ \\
\hline Blastocystis hominis + Dientamoeba fragilis & $\mathbf{2}$ & $\mathbf{1 . 2 5 \%}$ \\
\hline Entamoeba coli + Dientamoeba fragilis & $\mathrm{I}$ & $\mathbf{0 . 6 \%}$ \\
\hline Entamoeba coli + Giardia duodenalis & $\mathrm{I}$ & $\mathbf{0 . 6 \%}$ \\
\hline Entamoeba coli + Giardia duodenalis + Endolimax nana & $\mathrm{I}$ & $\mathbf{0 . 6 \%}$ \\
\hline Endolimax nana + Dientamoeba fragilis & $\mathrm{I}$ & $\mathbf{0 . 6 \%}$ \\
\hline
\end{tabular}

20 più protozoi non patogeni

e I elminta o protozoo patogeno (6.3\%)

${ }^{\circ}$ Entamoeba histolytica/dispar è stata interpretata come commensale, potenzialmente patogeno.

Tabella 4. Correlazioni tra parassitosi intestinali e quadro clinico*

\begin{tabular}{|c|c|c|c|c|c|c|c|c|}
\hline & $\mathrm{N}^{\circ}$ & Ipereosinofilia & $\begin{array}{c}\text { dolori } \\
\text { addominali }\end{array}$ & $\begin{array}{l}\text { disturbi intestinali } \\
\text { aspecifici }\end{array}$ & diarrea & $\begin{array}{c}\text { prurito } \\
\text { anale }\end{array}$ & $\begin{array}{c}\text { prurito } \\
\text { generalizzato }\end{array}$ & $\begin{array}{c}\text { nessun } \\
\text { sintomo }\end{array}$ \\
\hline PROTOZOI & 100 & $19 \%$ & $\mathbf{2 7} \%$ & $23 \%$ & $15 \%$ & $13 \%$ & $23 \%$ & $46 \%$ \\
\hline D. fragilis & 7 & $42.8 \%$ & $71.4 \%$ & $57.1 \%$ & $14.2 \%$ & $14.2 \%$ & $28.5 \%$ & $14.2 \%$ \\
\hline G. duodenalis & 9 & $11.1 \%$ & $11.1 \%$ & $22.2 \%$ & $11.1 \%$ & $22.2 \%$ & $33.3 \%$ & $55.5 \%$ \\
\hline B. hominis & 35 & $11.4 \%$ & $40 \%$ & $25.7 \%$ & $25.7 \%$ & $11.4 \%$ & $22.8 \%$ & $48.5 \%$ \\
\hline Altri non patogeni & 49 & $22 \%$ & $14.2 \%$ & $16.3 \%$ & $8.1 \%$ & $12.2 \%$ & $20.4 \%$ & $46.9 \%$ \\
\hline ELMINTI & 6 & $50 \%$ & $16.6 \%$ & $0 \%$ & $33.3 \%$ & $16.6 \%$ & $16.6 \%$ & $\mathbf{5 0 \%}$ \\
\hline
\end{tabular}

*Le percentuali riportate sono calcolate sul numero di soggetti che riferivano almeno un sintomo; i soggetti che riferivano diverse sintomatologie sono stati considerati più volte.

Tabella 5. Consistenza dei campioni fecali risultati positivi all'esame coproparassitologico standard

\begin{tabular}{cccccc}
\hline & $\mathrm{N}^{\circ}$ & Solida & Semisolida & Poltacea & Liquida \\
\hline PROTOZOI & $\mathbf{1 0 3}$ & $\mathbf{3 5 . 0 \%}$ & $\mathbf{4 3 . 6 \%}$ & $\mathbf{1 8 . 4 \%}$ & $\mathbf{3 . 0 \%}$ \\
\hline D. fragilis & 7 & $0 \%$ & $71.4 \%$ & $28.6 \%$ & $0 \%$ \\
\hline G. duodenalis & 9 & $44.5 \%$ & $22.2 \%$ & $22.2 \%$ & $\mathbf{1 1 . 1 \%}$ \\
\hline B. hominis & 35 & $11.5 \%$ & $62.8 \%$ & $22.8 \%$ & $2.9 \%$ \\
\hline Altri non patogeni & 52 & $53.8 \%$ & $30.8 \%$ & $13.4 \%$ & $2.0 \%$ \\
\hline ELMINTI & $\mathbf{6}$ & $\mathbf{8 3 . 3} \%$ & $\mathbf{0 \%}$ & $\mathbf{1 0 \%}$ & $\mathbf{0} \%$ \\
\hline
\end{tabular}

\section{RINGRAZIAMENTI}

Gli autori desiderano ringraziare la Dott.ssa Mara Manghi, Rossano Fornaciari e tutto il personale del "Centro per la Salute della Famiglia Straniera" di Reggio Emilia; il Dr. Claudio Dotti e il personale del Laboratorio Urgenze dell'Ospedale Santa Maria Nuova di Reggio Emilia per l'esecuzione degli esami emocromocitometrici. Si ringrazia inoltre il Dr. Daniele Crotti per la revisione scientifica del manoscritto.

Un ringraziamento particolare ai soggetti immigrati, senza la disponibilità dei quali non sarebbe stato possibile lo studio epidemiologico oggetto di questo manoscritto. 


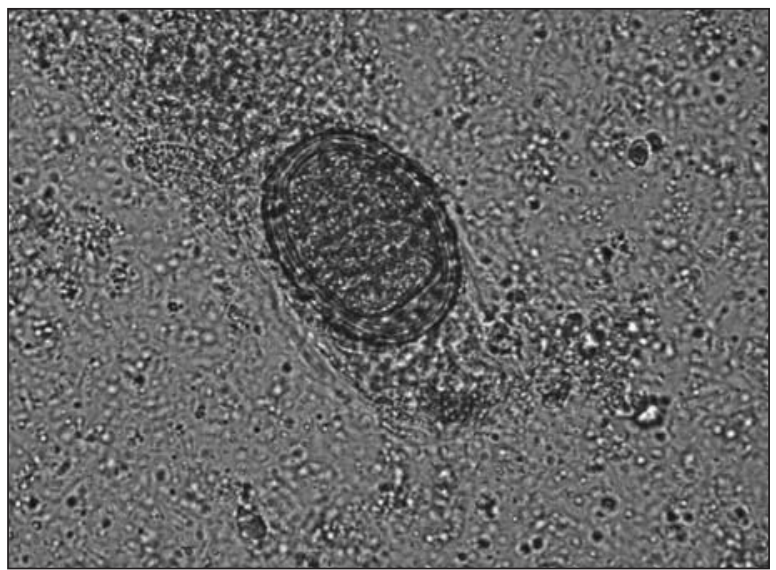

Figura II. Uovo di Ascaris lumbricoides (40X10) repertato dopo FEA.

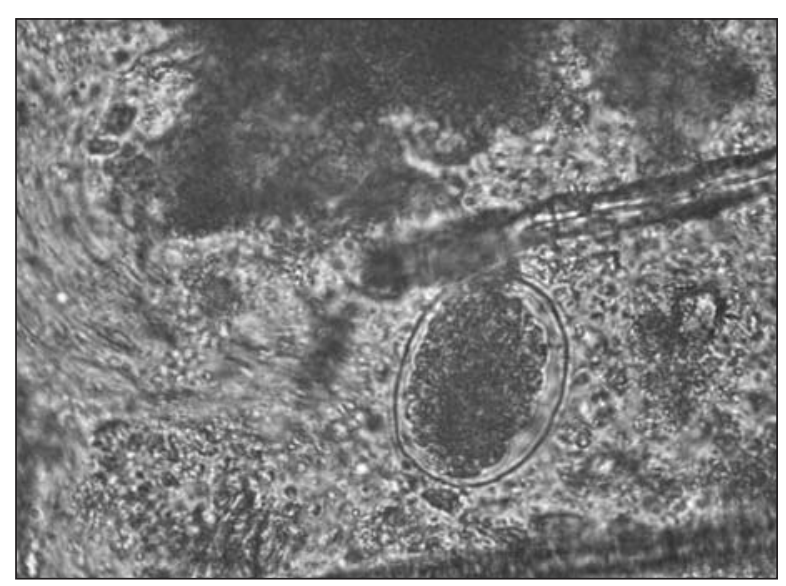

Figura III. Uovo di Ancylostoma duodenale/ Necator americanus (40XI0) repertato dopo FEA.

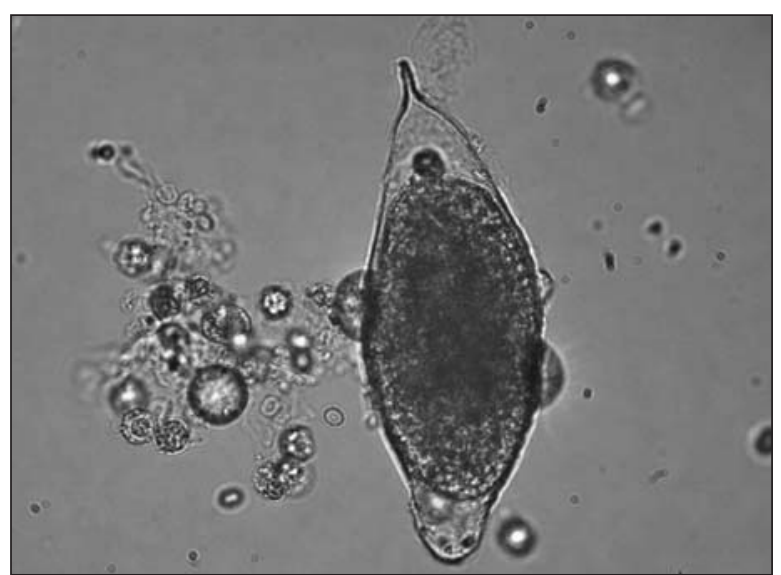

Figura IV. Uovo di Schistosoma haematobium (40X I0) repertato dopo FEA.

\section{BIBLIOGRAFIA}

1. Antico A, Pellizzer G, Romeo I, Corà R. Due casi di amebiasi nell'Ovest Vicentino: valutazioni epidemiologiche e cliniche. Microbiol Med 1998; 13: 351-3.

2. Baldo ET, Belizario VY, De Leon WU, Kong HH, Chung DI. Infection status of intestinal parasites in children living in residential institutions in Metro
Manila, the Philippines. Korean J Parasitol 2004; 42: 67-70.

3. Barbaro GB, Cavallino AM, Gianotti A, Patrone C, Robello A. Esami coproparassitologici: analisi di una casistica quinquennale raccolta nel territorio del Ponente Genovese; GIMMOC 2001; 3: 18-20.

4. Bernieri F, Crotti D, Galli D, Raglio A. Manuale illustrato di diagnostica parassitologica. Pavia: Edizioni Selecta Medica 2001.

5. Bernieri F, Galli D, Giordano S, et al. Indagine nazionale AMCLI-CoSP sulla diffusione delle parassitosi intestinali. Microbiol Med 1994; 9: 415-8.

6. Bernieri F, Galli D, Giordano S, et al. Indagine nazionale AMCLI-CoSP sulla diffusione delle parassitosi intestinali. Microbiol Med 1996; 11 (4): 463-72.

7. Bossi L, Perego L. Infezione da Cryptosporidium spp. in soggetti HIV positivi: una casistica. Microbiol Med 1991; 6: 81-4.

8. Cheesbrough M. Medical Laboratory Manual for Tropical Countries; Vol I, Butterworth Ed 1987.

9. Cimerman S, Ladeira MC, Iuliano WA. Blastocystosis: nitazoxanide as a new therapeutic option. Rev Soc Bras Med Trop 2003; 36: 415-7.

10. Crotti D, Bernieri F, Raglio A, Galli D. L'esame copro parassitologico: indicazioni di base per una adeguata diagnostica. Rivista della Società Italiana di Medicina Generale 2005; 5/6: 28-32.

11. Crotti D, D’Annibale ML, Medori MC, Fonzo G, Ubaldi M. Parassitosi intestinali autoctone nell'area del Perugino: considerazioni di metodologia diagnostica e prevalenze nel corso del 2001. GIMMOC. 2003; VII (1): 12-20.

12. Crotti D, D’Annibale ML. Diagnostica di epidemiologia delle infezioni intestinali umane nel territorio perugino nel corso del 2003. Bollettino di microbiologia e indagini di laboratorio news 2004; 10: 7-15.

13. Crotti D. Le parassitosi intestinali ed uro-genitali conoscenze di base e indicazioni diagnostiche. Caleidoscopio Italiano 2006 Ed. Selecta medica.

14. D’Annibale ML, Bracciale S, Vitali M, Fonzo G, Verdini C, Papili R. Parasitic intestinal infections in humans between 2006 and 2007. Microbiol Med 2009; 4: 19-24.

15. D’Annibale ML, Crotti D. Parassitosi intestinali umane nel perugino nel corso del 2005. Microbiol Med 2007; 22: 57-65.

16. De Carneri I. Parassitologia Generale e Umana; XI Ed., Casa Editrice Ambrosiana, Milano 1992.

17. Garcia LS. Practical Guide to Diagnostic Parasitology. American Society for Parasitology, Washington DC 1999.

18. Garcia LS, Shimizu RY, Bernard CN. Detection of Giardia lamblia, Entamoeba histolytica/dispar, and Cryptosporidium parvum antigens in human fecal specimens using the Triage parasite panel enzyme immunoassay. J Clin Microbiol 2000; 38: 3337-40.

19. Guidetti C, Capatti C, Ricci L, Vecchia L. Parassitosi intestinali nell'area reggiana nel corso del 2007: analisi critica dei risultati. Microbiol Med 2009; 24 (2): 103-7.

20. Johnson EH, Windsor JJ, Graham Clark C. Emerging from Obscurity: Biological, Clinical, and Diagnostic Aspects of Dientamoeba fragilis. CMR 2004; 17: 55370.

21. Lainson R, da Silva BAM. Intestinal Parasites of some diarrhoeic HIV-positive individuals in North Brazil, with particular reference to Isospora belli Wenyon, 1923 and Dientamoeba fragilis Jepps \&Dobell 1918. Mem Inst Oswaldo Cruz 1999; 94 (5): 611-3.

22. NCCLS. Procedures for the Recovery and 
Identification of Parasites from the Intestinal Tract; Approved Guideline. M 28-A. NCCLS, Waine PA USA 1997.

23. Nocera E, Bozzelli L, Gallo MA, et al. Epidemiologia delle parassitosi intestinali in una popolazione di immigrati extracomunitari. Microbiol Med 2006; 4: 328-32

24. Norberg A, Nord CE, Evengaard B. Dientamoeba fragilis a protozoal infection which may cause severe bowel di stress. CMI 2003; 9: 65-8.

25. Pampiglione S, Canestri Trotti G. Guida allo studio della Parassitologia. Società Editrice Esculapio, Bologna,. parasitism in a cohort of Peace Corps volunteers in Guatemala. J Clin Microbiol 1990; 39: 3442.

26. Peruzzi S, Gorrini C, Piccolo G, Calderaro A, Dettori G, Chezzi C. Prevalence of Intestinal parasites in the area of Parma duringe the year 2005. Acta Biomed 2006; 77: 147-51
27. Petithory JC, Ardoin-Guidon F, Chaumeil C. Amibes et flagelles intestinaux. Amibes oculaires. Leur diagnostic microscopique. Bioforma, Paris France1988. Cahier de Formation, Biologie medicale no. 11.

28. Rondanelli EG, Scaglia M. Atlante dei protozoi umani. Masson Ed, Milano 1993.

29. Scaglia M, Gatti S, Rondanelli EG. Parassiti e parassitosi umane, dalla clinica al laboratorio. Pavia: Edizioni Selecta Medica 2006.

30. Stark D, Beebe N, Marriot D, Ellis J, Harkness J. Detection of Dientamoebiasis: clinical importance and recent advances. TRENDS in Parasitology 2006; 22: 92-6.

31. WHO. Basic Laboratory Methods in Medical Parasitology. WHO, Geneva CH 1991.

32. WHO. Bench Aids for the Diagnosis of Intestinal Parasites. WHO, Geneva 1994.

33. Zeibig EA. Clinical Parasitology. A pratical Approach. Saunders, Philaledlphia. 1997. 